\title{
CORRECTION
}

\section{Correction: Sex-specific alterations in preterm brain}

Amanda Benavides ${ }^{1}$, Andrew Metzger ${ }^{2}$, Alexander Tereshchenko ${ }^{1}$, Amy Conrad $^{3}$, Edward F. Bell ${ }^{3}$, John Spencer ${ }^{4}$, Shannon Ross-Sheehy ${ }^{5}$, Michael Georgieff ${ }^{6}$, Vince Magnotta ${ }^{2}$ and Peg Nopoulos ${ }^{1,3,7}$

Pediatric Research (2019) 85:732; https://doi.org/10.1038/s41390-019-0323-x

Correction to: Pediatric Research (2019) 85:55-62; https://doi.org/ 10.1038/s41390-018-0187-5; published online 19 September 2018

In the original article, the legend within Fig. 3 incorrectly read as ${ }^{* *} p<0.10,{ }^{* *} p<0.05,{ }^{* *} p>0.01$ '. This has now been changed to
${ }^{* *} p<0.10,{ }^{* *} p<0.05,{ }^{* * *} p<0.01$ '. This has been corrected in both the PDF and HTML versions of the Article. The authors would like to apologise for this error.

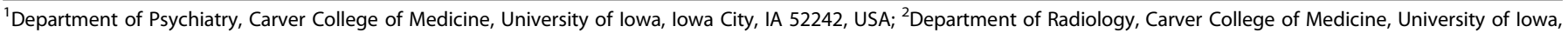

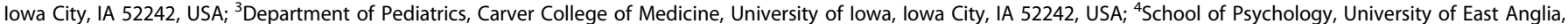

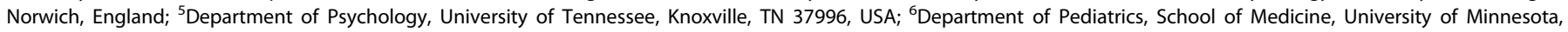
Minneapolis, MN 55455, USA and ${ }^{7}$ Department of Neurology, Carver College of Medicine, University of lowa, lowa City, IA 52242, USA

Correspondence: Peg Nopoulos (peggy-nopoulos@uiowa.edu)

These authors contributed equally: Amanda Benavides, Andrew Metzger

Published online: 8 February 2019 